\title{
Evaluating the Human Damage of Tsunami at Each Time Frame in Aggregate Units Based on GPS data
}

\author{
Y. Ogawa ${ }^{\text {a, }}$, Y. Akiyama ${ }^{\text {b }}$, H. Kanasugi ${ }^{\text {c }}$, R. Shibasaki ${ }^{\text {b H. Kaneda }}{ }^{\mathrm{d}}$ \\ ${ }^{\text {a }}$ Graduate School of Frontier Science, The University of Tokyo, 4-6-1 Komaba, Meguro-ku, Tokyo, Japan- \\ ogawa@csis.u-tokyo.ac.jp \\ ${ }^{\mathrm{b}}$ Center for Spatial Information Science, The University of Tokyo, 5-1-5 Kashiwanoha, Kashiwa city, Chiba, Japan- \\ aki@iis.u-tokyo.ac.jp, shiba@csis.u-tokyo.ac.jp \\ ${ }^{c}$ Earth Observation Integration \& Fusion Research Institute, The University of Tokyo, 4-6-1 Komaba, Meguro-ku, Tokyo, Japan- \\ yok@iis.u-tokyo.ac.jp \\ ${ }^{\mathrm{d}}$ ZENRIN DataCom Co.LTD , Shinagawa Intercity Tower C 6F, 2-15-3 Konan, Minato-ku, Tokyo - \\ h_kaneda@zenrin-datacom.net
}

\section{Commission VIII, WG VIII/1}

KEY WORDS: GIS, The Nankai trough megathrust earthquake, Evacuation, Casualties, Population distribution

\begin{abstract}
Assessments of the human damage caused by the tsunami are required in order to consider disaster prevention at such a regional level. Hence, there is an increasing need for the assessments of human damage caused by earthquakes. However, damage assessments in japan currently usually rely on static population distribution data, such as statistical night time population data obtained from national census surveys. Therefore, human damage estimation that take into consideration time frames have not been assessed yet. With these backgrounds, the objectives of this study are: to develop a method for estimating the population distribution of the for each time frame, based on location positioning data observed with mass GPS loggers of mobile phones, to use a evacuation and casualties models for evaluating human damage due to the tsunami, and evaluate each time frame by using the data developed in the first objective, and 3) to discuss the factors which cause the differences in human damage for each time frame. By visualizing the results, we clarified the differences in damage depending on time frame, day and area. As this study enables us to assess damage for any time frame in and high resolution, it will be useful to consider provision for various situations when an earthquake may hit, such as during commuting hours or working hours and week day or holiday.
\end{abstract}

\section{INTRODUCTION}

In recent years, Tsunami will hit hard in Japan by a Nankai trough megathrust earthquake with a scale of at least Mm.9. In addition, in August 2014, the investigation committee of the Japanese government has for the first time announced the assumed height of the tsunami in the Sea of Japan (Ministry of land, infrastructure and transport, 2014). For the tsunami that has been predicted with a high risk in Japan, quantitatively estimate the building damage, to assess the human and material risk in advance. It is important for considering the national land and regional disaster management policy. A Company have to know how much of the damage is expected when planning BCP (Business Continuity Plan) for the location plan of the company is essential to the company resilience improvement. For disaster mitigation, the Japanese government is to determine a basic plan for advancing disaster prevention measures in the case of a Nankai trough megathrust earthquake. Developing an environment for evaluating and comparing earthquake disaster risks for specific regions with high accuracy is strongly required

When estimating the human casualties caused by the tsunami we use the evacuation model and the tsunami fragility function. Fragility function is a formulating the relationship between the external force such as inundation depth and flow and damage ratio. Many damage assessments in Japan have been used fragility function which estimate houses and human damage ratio by using the inundation depth and flow rate as explanatory variables by the 2004 Sumatra earthquake tsunami (Koshimura et al., 2009). On the other hand, Ogawa et al. (2015) develop tsunami fragility carve for evaluate the dead ratio and building collapse ratio in the 2011 Tohoku earthquake tsunami by using the data which conducted on the flooded area field surveyed by the Ministry of Land, Infrastructure and Transport after the disaster. The data is reliable, because the data included all building damage information (disaster situation and the inundation depth) of inundation area. In addition, We have created a capable database by using fragility carve to estimate the building damage of the building in each building by giving any of inundation depth throughout Japan, and that can grasp relative risk assessment in the inter-regional.

However, essential data for estimating precise damage by earthquakes in Japan is not yet prepared or fully open to the public. Human damage estimation that takes time frames into consideration is necessary, yet population distributions depending on time frames have not been developed. Current damage assessments in Japan usually rely on static population distribution data, such as night time population census, organized in $250 \mathrm{~m}$ grid units obtained from national census. Considering disaster prevention policies, it is important to develop population distribution which can evaluate disaster risk in a variety of units, from macro scale such as prefectures to micro scale such as city block units.

Against this background, when performing damage assessment and forming evacuation plans for catastrophic

\footnotetext{
* Corresponding author
} 
earthquakes and tsunamis, it is essential to have data that can grasp the detailed location information of people distributed in cities for each time frame. In order to understand the congestion and flow of people for each time frame, the National PersonTrip (PT) Survey, which is a kind of traffic survey data, has been used (Ogawa et al., 2015). Ogawa et al., 2015 survey examines the actual stationary travel patterns of people living in each survey area, and its objective is to figure out "who" travels "from where to where" "when" "for what purpose" and "by which mode of transportation". Since the Person-Trip Survey is a questionnaire, representative of only a part of the actual population, a magnification coefficient is allotted to each dataset according to the actual population, and data is aggregated for each survey area. Although PT data can solve many of the problems described above to understand the distribution and flow of people in a city, often the survey area becomes larger in rural areas and overseas. Since the positions of origins and destinations in PT data is typically represented by the center of a survey area (zone), when mapping PT data on a map the population of that area becomes focused on that center point and the data becomes biased. To solve such problems, Ogawa et al., (2015) clarify the spatial definition of the position information of PT data, based on the distribution of buildings. There are studies about spatial definition for each time frame based on the actual position information of buildings that consider the magnification factor in such a wide area. But PT data can only grasp on weekdays and the update once in 10 years, so the PT data cannot consider in holiday and long-term movement of people.

In recent years, the spread of the GPS equipped mobile phones in the world, a large Geo spatial data are accumulated daily on the movement log of the person represented by big data. The data are expected to be able grasp the flow of people because of the accumulation of mobile phone device data in Japan. By using mobile phone data in previous studies, Ye et al., (2009) grasp behavioral patterns of the each user. Also the Tourism Agency (2015) grasps the dynamics of tourists from the behavior by using the location information from GPS data.

On the other hand, previous studies have not seen that the use of large-scale GPS data in disaster management. This is because the data is not easily to obtain and it takes time of calculation. Moreover, when applying to a disaster, it is necessary to add a magnificent coefficient to match the actual population for a sample-based and identify whether the person is moving or are staying. In this study, our objective is to estimate of the population in each time frame with a mobile phone GPS data and using them to estimate the human damage by the tsunami. Also by using the latest damage calculation method of the Japanese government, we estimate the human damage by applying the GPS data of the October 2010. Finally, In combination with evacuation setting scenario (early evacuation, after tasks-evacuation) and the population data make the multiple scenarios and we discuss by statistical analysis of the results. In order to evaluate damage for each time frame for tsunamis, implementation of this technique is important. By realizing this method, risk assessment for any time frame and any aggregate unit will be possible and lead to the optimal decision-making for disaster management. On a municipal scale, it is important for advancing urban planning and development in times of disaster. At the residential level, disaster prevention awareness is important for decision-making, such as overall improvement as well as time frame based analysis for each evacuation plan.

\section{DEVELOPMENT}

\subsection{Flow of the evaluating the human damage of Tsunami} at each time frame

There are three main advantages to our method. First, we can compare damage evaluation for many time frames and day, instead of just nighttime and daytime or weekdays and holidays. Second, high-definition population data enables us to evaluate casualties in high-spatial resolution. Third, our method can evaluate the risk of fatalities from Tsunami caused by the Nankai trough megathrust earthquake, for any aggregate unit from $5 \mathrm{~m}$ grid unit, considering the ground characteristic and properties of each grid (elevation, land use, etc.). Figure 1 shows the processing flow of our method. First, to perform the evaluation for each timeframe, we use the GPS equipped mobile phones for a processed GPS data (the GPS data are processed with individual concealment by NTT Docomo, INC. and provided by ZENRIN DataCom Co., LTD.) to estimate each time frame population. Second, we estimate population distribution in each time frame from GPS data by adding magnificent number by using the population census and the economic census. Third, calculate number of casualties in each grid ( $5 \mathrm{~m}$ grid), we join the simulation data of Nankai trough megathrust earthquake tsunami provided by International Research Institute for Disaster Science, Tohoku University to clarify the estimated the tsunami arrival time and height in 30 second interval for 180 minutes after the earthquake hits, and calculate evacuation risk based on a few scenarios (setting ratio of the direct evacuees and the evacuees after errand). Finally, we estimate the damage assessments of humans, such as the number of casualties, by combining population distribution in each time frame and each grid's evacuation risk. This amount can be aggregated to $250 \mathrm{~m}$ grid unit to get the numbers of casualties for each time frame.

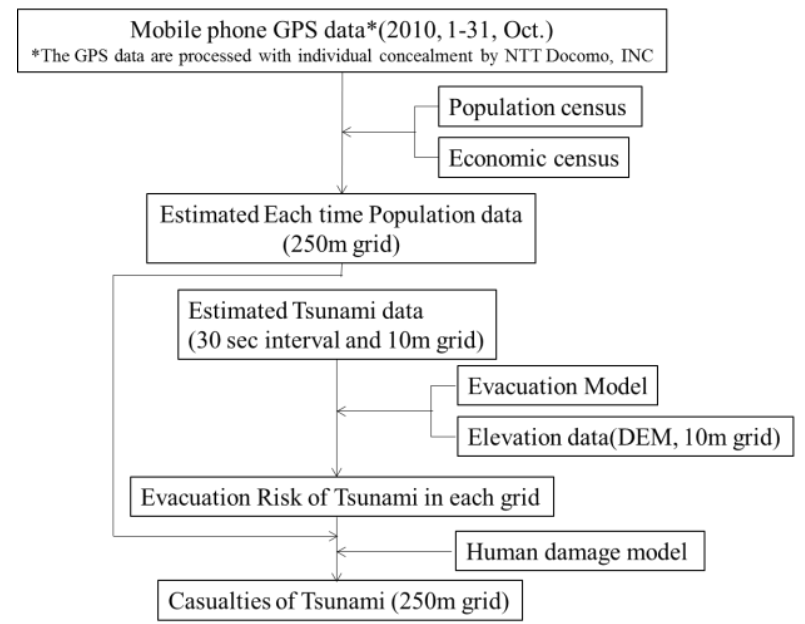

Figure 1, Flow chart of estimating causalities

\subsection{Development of estimation of population based on mobile phone GPS data.}

In this study, we use aggregated data of mobile phone GPS logs in 31 days in 2012, October called the "Congestion Analysis ${ }^{\circledR}$ " provided by ZENRIN DataCom Co. LTD. The source data of the Congestion Analysis ${ }^{\circledR}$ are disaggregated data of mass people flows collected by the auto-GPS function on mobile phones of NTT Docomo (NTT Docomo, INC., 2009). The data 
was obtained from phone users who permit to be used their information collected by mobile phone carriers. These data do not reveal any information about an individual. The movement history of a specific individual cannot be monitored by the security processing. This is a large database that contains about 1.5 million users constructed from text data of approximately 9 billion records.

First, the GPS data was performed a pre-processing as an extraction of the stay point of the user from each position coordinates and observation time. The stay point defines that the representative point of a user remains a certain time and in a certain range. In this study, more than 15 minutes and the radius of $300 \mathrm{~m}$ as a condition of stay, and the central point of maximum rectangle from the column of points of staying period to be the stay points (Horanont (2010); Horanont et al. (2013); Hadano (2013)). Also this data holding time information of the arrival time and the departure time for each stay point. In addition, we estimated the work location and the home location to each data from stay point and the observation time. In addition, in order to match the GPS data on population, the estimation of the magnification coefficient from the estimated work location and home location. The estimation of the magnification coefficient, we used the statistical data of the number of the population and employees. We use the 250mmesh data of population census 2010 for residential population and the $250 \mathrm{~mm}$ esh of economic census 2010 for the number of employees.

Figure 2 shows a method of estimating the magnification number. Magnification number estimated by dividing the residential population by the number of terminals at home place in 250mmesh unit $(\mathrm{Mh})$ and estimate magnificent coefficient (Mw) by dividing the number of employees by the number of terminals at the work location. From the average of the two magnificent coefficients ( $\mathrm{Mh}$ and $\mathrm{Mw}$ ) is the final magnificent coefficient $M$. Figure 3 is the estimated time a different population that granted the expansion coefficient to the GPS data. By imparting magnification factor to each data became possible to estimate the population distribution of the population based rather than population distribution of a sample basis.

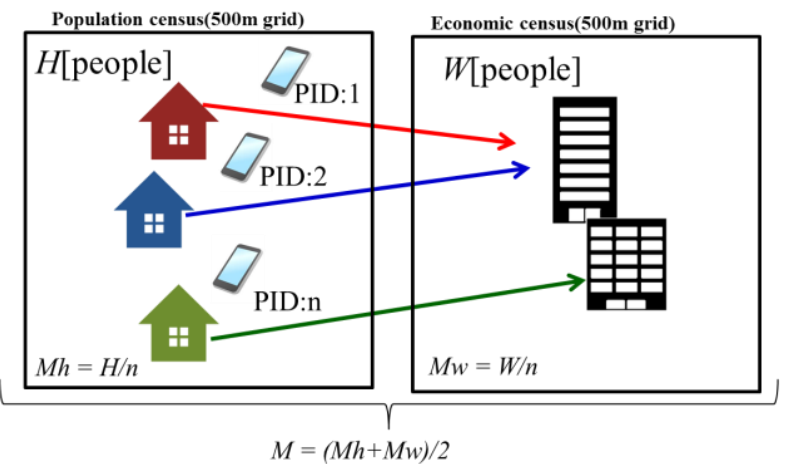

Figure 2, Method of estimating magnificent number (people/ a phone)

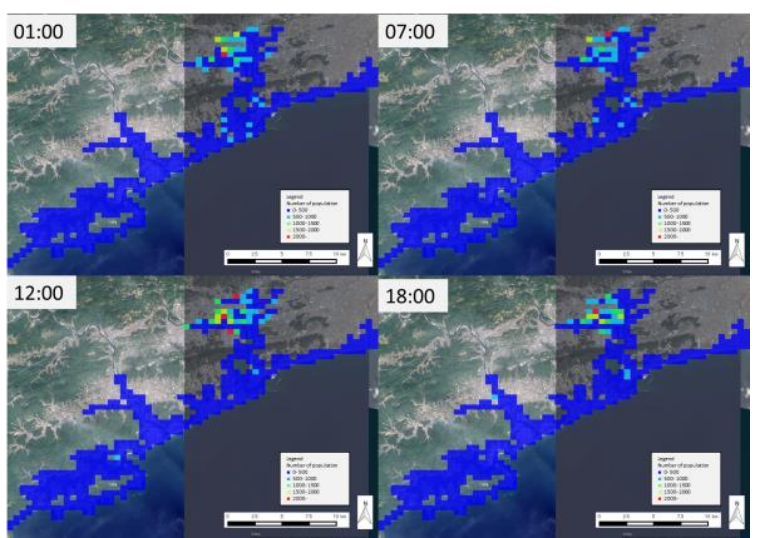

Figure 3, Number of population in study area (2010, 01, Oct.)

\subsection{Reliability verification of a magnification coefficient}

The reliability of our data is verified by aggregated to $250 \mathrm{~m}$ grid (the local community unit in general) and to compare the number of home place and work place population throughout Japan. The verification data uses $250 \mathrm{~m}$ grid-cell data of Population census (2010) and Economic census (2010). Figure 4 shows comparison results for home place population. There is strong correlation of $\mathrm{R}=0.96$ between the numbers of home place population from our data with the actual numbers of population census. On the other hand work place population has also has strong correlation of $\mathrm{R}=0.94$ between the number of economic census (figure 5). As a result, our estimated magnification coefficient data is reliable for evaluating performances in each local community.

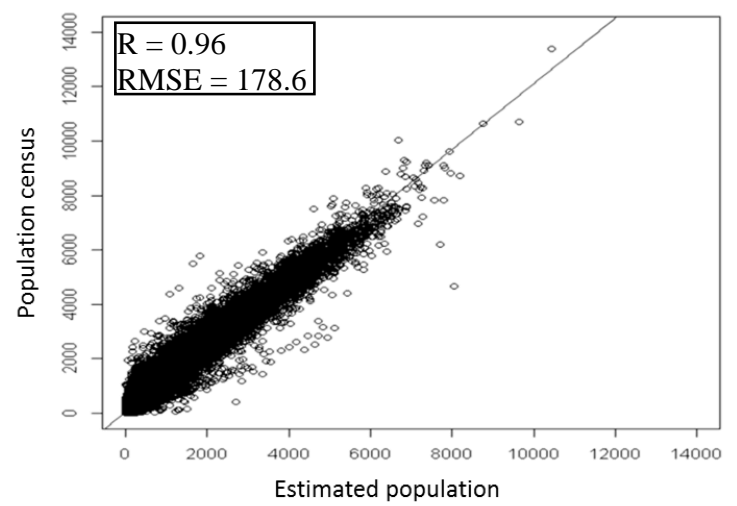

Figure 4, Correlation between the numbers of home place population in our data with the population census

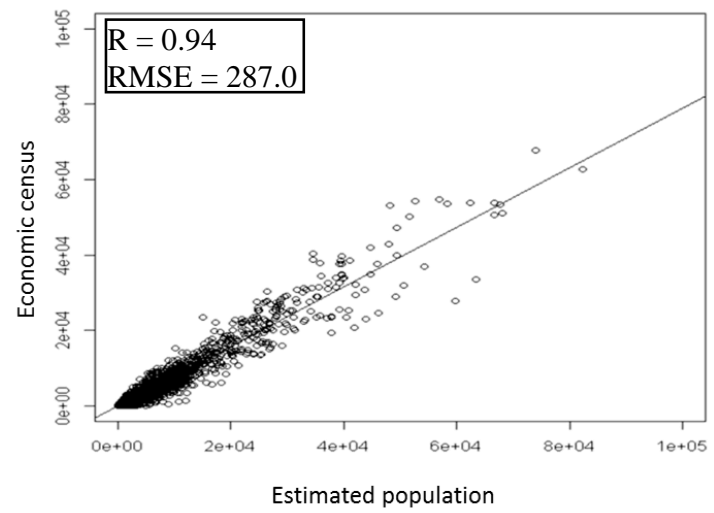

Figure 5, Correlation between the numbers of work place population in our data with the economic census. 


\section{DAMAGE MODEL OF TSUNAMI}

\subsection{Evacuation model of tsunami}

About input data of tsunami, we use numerical simulation results assuming the Nankai trough megathrust earthquake provided from International Research Institute for Disaster Science, Tohoku University. Figure 6 shows the maximum inundation depth of the numerical simulation result. The numerical simulation results include immersion depth data of 3 hours of 30 seconds interval after disaster $(10 \mathrm{~m}$ mesh). The total numbers of scenarios are 24 hours * 31 days (2010, October) * 3 types of evacuation behaviour pattern: 2232 scenarios in a total.

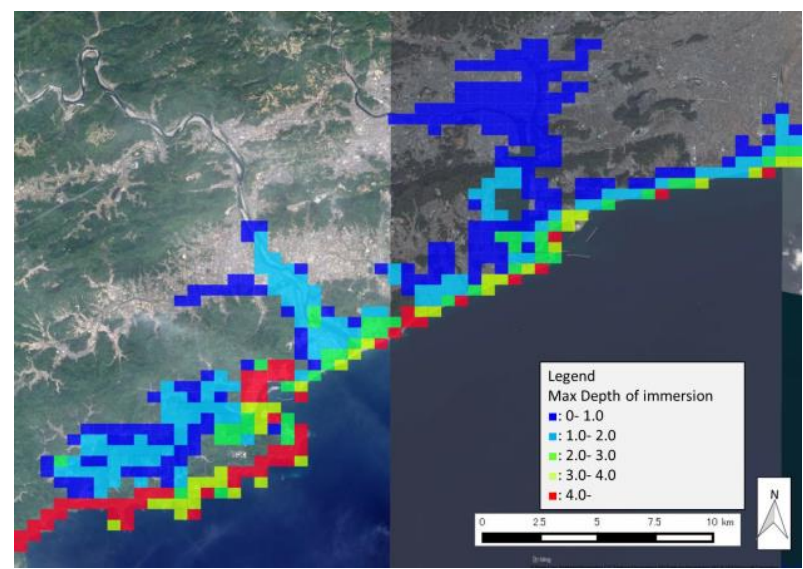

Figure 6, The maximum inundation depth in study area

\subsubsection{The difference between evacuation behaviour}

Evacuation patterns are shown in the table 1 according to Great East Japan Earthquake Survey results in the affected region of "the Survey results for the tsunami evacuation" (Cabinet Office, the Fire and Disaster Management Agency, the Japan Meteorological Agency, 2013).

Table 1, Setting of evacuation behaviour

\begin{tabular}{|c|c|c|c|}
\hline \multirow[t]{3}{*}{ Scenario } & \multicolumn{3}{|c|}{ Ratio of each evacuation action [\%] } \\
\hline & \multicolumn{2}{|c|}{ Evacuate } & \multirow{2}{*}{$\begin{array}{l}\text { Not or urgency } \\
\text { evacuate }\end{array}$} \\
\hline & Immediately & $\begin{array}{c}\text { Not } \\
\text { immediately }\end{array}$ & \\
\hline No. 1 & 100 & 0 & 0 \\
\hline No. 2 & 70 & 30 & 0 \\
\hline No. 3 & 70 & 20 & 10 \\
\hline
\end{tabular}

\subsubsection{Evacuation uncompleted rate}

The proportion of people, who cannot complete evacuation from stay point to a safe place at the time of the disaster, was calculated by the following method showing in figure 7 (Central Disaster Management Council, 2012).

i) Specific grid of requirements evacuation

Maximum tsunami inundation depth has identified the essential evacuation mesh equal to or larger than $30 \mathrm{~cm}$.

ii) Estimating of grid of evacuation destination

Located in the shortest distance from each main evacuation grid (grid of evacuation origin) and the tsunami inundation depth $1 \mathrm{~cm}$ arrival time than the grids of evacuation origin are identified grid of evacuation destination of less than tsunami inundation depth $30 \mathrm{~cm}$.

iii) Evacuation distance is 1.5 times of the Euclidean distance between centers of grid. (The Great East Japan Earthquake of performance (Cabinet Office, 2013)). iv) The calculation of the evacuation time required to complete for each evacuation grid, to calculate the required evacuation time to complete divided the evacuation distance by evacuation speed (the average speed $2.65 \mathrm{~km} /$ hour from the Great East Japan Earthquake of results). It should be noted that the evacuation start time at daytime disaster, disaster after 5 minutes in the direct evacuees, and after 15 minutes on the evacuees after errand, urge evacuees to evacuate from tsunami in the grid has reached.

v) The determination of evacuation success or failure

For each main evacuation grid, we compare the evacuation time required to complete the inundation depth $30 \mathrm{~cm}$ arrival time in the adjacent grid of refuge grid and to shelter mesh, then determined to evacuate the success or failure by evacuation person. The determination calculation evacuation completed and uncompleted was carried out as follows.

In the case of the night, evacuation started with the assumption that take a long time to a further 5 minutes preparation than in the daytime, evacuation rate was also assumed to decline to $80 \%(2.09 \mathrm{~km} /$ hour $)$ of the daytime.

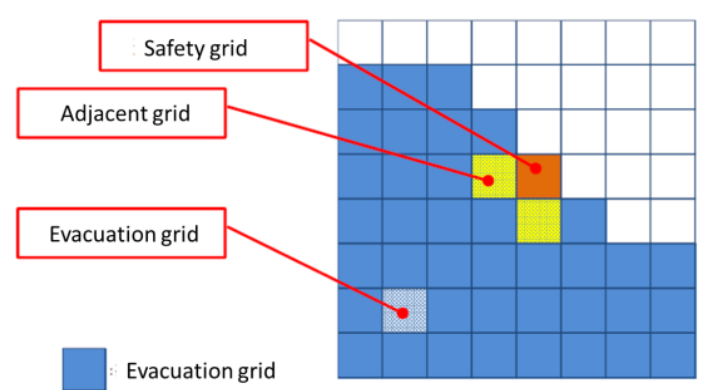

Figure 7, Relation of Evacuation grid and Safety grid

\subsection{Model of estimating for human casualties}

3.3 In each of the required evacuation mesh, to calculate the number of deaths by using, fragility functions with respect to evacuation incomplete person (deaths occurred in inundation depth $30 \mathrm{~cm}$ or more and all deaths in the depth of $1 \mathrm{~m}$, immersion cumulative distribution function of the normal distribution: figure 8).

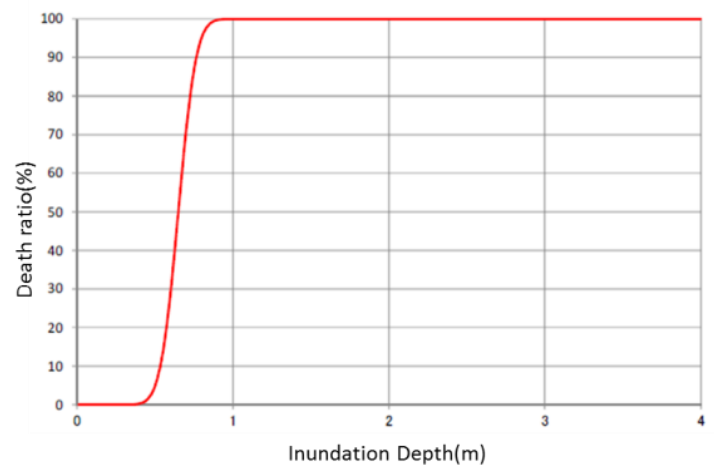

Figure 8, Fragility carve of casualties (Central Disaster Management Council, 2012)

\section{RESULT}

Figure 9 shows the result of the number of human casualties evaluation in each time frame (when using GPS data of 1 Oct., 
2010) caused by the tsunami in the event of the Nankai trough megathrust earthquake. The target area of the result was Kochishi in Kochi prefecture and we input estimated tsunami height for each $5 \mathrm{~m}$ grid-cell.in the targeted area. The result shows that our method can evaluate the damage for each time frame in high spatial resolution. The result reveals that damage situation in some area depends on the time frame. We visualized the result and clarified the differences in damage for each time zone and each region at scenario 2 (figure 9). In addition, we visualized the result and clarified the differences in damage for each day and each scenario. Figure 10 shows the result of the differences in each day at P.M.6. The damage depends on each time frame and on week day (Ex. 4-8 Oct., 2010) and holiday (Ex. 9 and 10 Oct., 2010) as people move around the city. Since earthquake assumed in this scenario is large, tsunami height and casualties vary greatly from late at night which people stay at their house. The damage is small at 8 A.M. because it is commute time zone and thus de facto population is small. This is the first data to input any expected seismic motion data and evaluate human fatalities in each time frame by considering de facto population.

Figure 12 shows histogram of total human casualties by the tsunami for all scenarios in the study areas. Although result indicates that the mode in the number of casualties following 1000 people is most frequently. Also in the number of casualties 5001-6000 is high frequency. Result indicating that not only the mode scenarios when the assumed damage, the larger scenario of such damage quantity is large frequency so we also should have considered. Figure 13 shows a scenario in which casualties' amount in the above-average value is the mode. It shows that the amount of damage is greater in a large population the city and close to the coast.

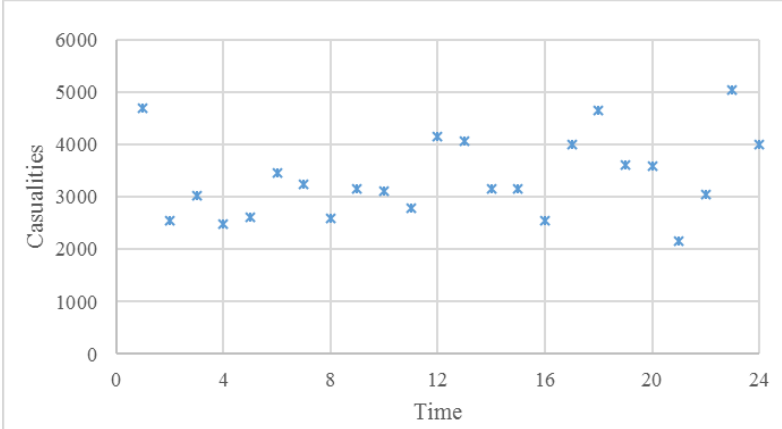

Figure 9, Number of casualties in each time frame in study area (scenario: 3, on 1 October, 2010)

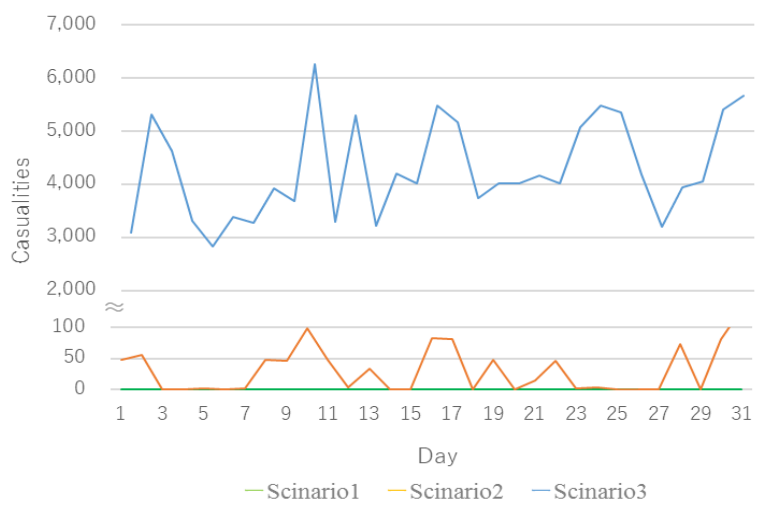

Figure 10, Number of casualties in each day and each scenario ( October, 2010)

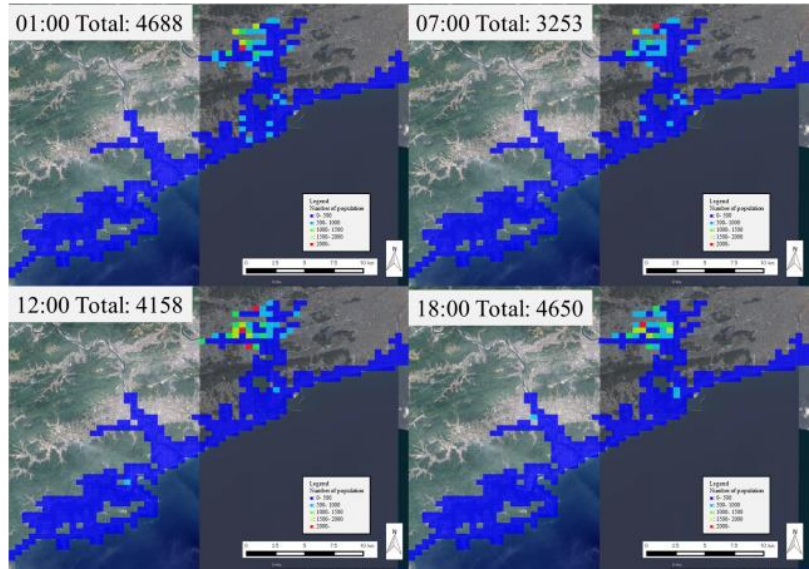

Figure 11, Evaluation of human casualties by tsunami on the Nankai trough megathrust earthquake in the each time frame at scenario 2 on 1 October, 2010 (aggregated to 500m grid units)

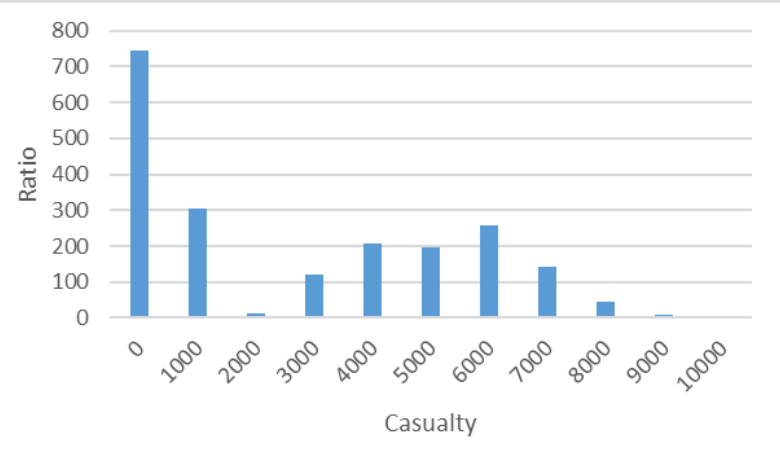

Figure 12, Total casualties and frequency in study area

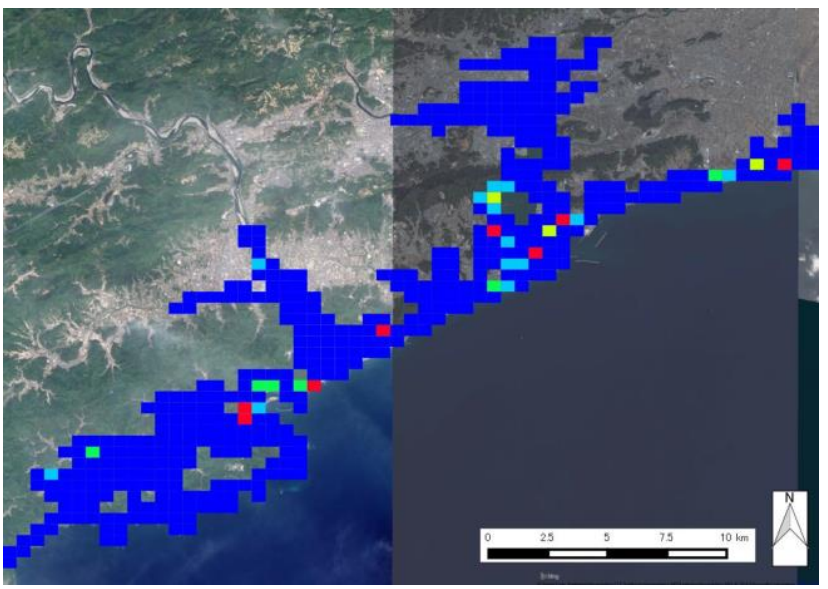

Figure 13, Mode value sinalio of casualties by tsunami on the Nankai trough megathrust earthquake (aggregated to $500 \mathrm{~m}$ grid units)

\section{CONCLUSION AND FUTURE WORK}

In this study, we estimated the detailed population distribution for each time frame by using mobile phone GPS data. In addition, we evaluated the possibility of tsunami taking into consideration the magnitude of earthquake by adding numerical simulation data of tsunami based on the Nankai trough earthquake to each $5 \mathrm{~m}$ grid-cell. By combining these data and population for each time frame, we evaluated human damage caused by the Nankai trough megathrust earthquake tsunami. Our method is unique in that it enables us to assess damage for 
any time frame and high spatial resolution, and thus it also enables us to evaluate human damage due to tsunami caused by earthquakes in broad areas. In the present study, the target area of damage evaluation was the Kochi-shi, Kochi prefecture, yet we also can evaluate other areas, for calculations of evaluation were set automatically. We have two issues as future outlook; firstly, we estimate magnificent number for GPS data only used night population data and work place population data obtained from the population census and economic census to estimate magnificent number of our population distribution. In addition there is such data of the station between the numbers of passenger of the train in Japan; it is possible to more reliable estimation of population data by utilizing them. This paper we only considering tsunami but building collapse by ground motion and fire are also scheduled to simulate for integrate assessment. In the future study, we will also consider building data usage and business hours in order to estimate with the high definition population distribution data of the person unit rather than grid unit.

\section{ACKNOWLEDGEMENTS}

We would like to thank Dr. Syunichi Koshimura, Professor of the International Research Institute of Disaster Science, Tohoku university for provide the tsunami data in this study. In addition, we would like to thank CSIS (the joint research No. 448) and ZENRIN DataCom Co.LTD for provide the Congestion Analysis ${ }^{\circledR}$.

\section{REFERENCES}

Central Disaster Management Council, 2012, About the Nankai Trough earthquake measures of building damage and human damage (in Japanese), Available at <

http://www.bousai.go.jp/jishin/nankai/taisaku/pdf/2_2.pdf>, [Accessed 15, April]

Hadano, M., Wiayangkurn, A., Akiyama, Y., Horanont, T. and Sibasaki, R., 2013, A Study on Extracting Characteristic of Visitors at Commercial Area from GPS Data, IGU 2013 Kyoto regional conference, 01435

Horanont, T., 2010. A Study on Urban Mobility and Dynamic Population Estimation by Using Aggregate Mobile Phone Sources. Department of civil engineering, The University of Tokyo., PhD. Thesis.

Horanont. T., Phithakkitnukoon, S., Leong, W, T., Sekimoto,Y. and Shibasaki, R., 2013. Weather Effects on the Patterns of People's Everyday Activities: A Study Using GPS Traces of Mobile Phone Users. PLOS ONE, 8(12), e81153.

International Research Institute for Disaster Science, Tohoku University, Available at:

<http://www.tsunami.civil.tohoku.ac.jp/hokusai3/J/index.html>, [Accessed 2015, 15, April]

Japan tourism agency, 2015, Tourism promotion utilizing tourism big data/ Research and analysis of tourist behavior using GPS, Available at

<https://www.mlit.go.jp/kankocho/shisaku/kankochi/gps.html>, [Accessed 2015, 15 April]

Koshimura S., Namegaya Y., Yanagisawa H., 2009, Fragility Functions for Tsunami Damage Estimation, Doboku Gakkai Ronbunshuu B, Vol. 65, No. 4, pp320-pp331
Ministry of Land, Infrastructure and Transport, 2012, About the placement and evacuation of tsunami evacuation and evacuation routes and evacuation facility

Ministry of land, infrastructure and transport, 2014, Report of survey study group on a large-scale earthquake in the Japan Sea

Ogawa Y, Akiyama Y, Sengoku H, Shibasaki R, 2013, Development for the eval-uation of Catastrophic Earthquake Damage throughout Japan Using Estimat-ed Micro Data. Computers in Urban Planning and Urban Management (CUPUM), conference papers 103

Ogawa Y, Akiyama Y, Sengoku H, Shibasaki R, Kato T, 2014, Development of Tsunami Fragility Curves and Evaluation of Damage due to the 2011 Tohoku Earthquake, CSIS DAYS, A03

Y. Ye, Y. Zheng, Y. Chen, J. Feng, and X. Xie, 2009. Mining Individual Life Pattern Based on Location History, Proc. Int'1. Conf. on Mobile Data Management Systems, Services and Middleware, 1- 10. 